\title{
BMJ Open Use of IMMPACT domains in clinical trials of acupuncture for chronic pain: a protocol for a methodological survey
}

\author{
Lauren Giustti Mazzei, Cristiane de Cássia Bergamaschi, Marcus Tolentino Silva, \\ Luciane Cruz Lopes
}

To cite: Mazzei LG, Bergamaschi CdC, Silva MT, et al. Use of IMMPACT domains in clinical trials of acupuncture for chronic pain: a protocol for a methodological survey. BMJ Open 2017;7:e014904. doi:10.1136/ bmjopen-2016-014904

- Prepublication history and additional material for this paper are available online. To view please visit the journal (http:// dx.doi.org/10.1136/bmjopen2016-014904).

Received 25 0ctober 2016 Revised 15 August 2017

Accepted 16 August 2017

\section{CrossMark}

Department of Pharmaceutical Sciences Graduate Program, University of Sorocaba, Sorocaba, São Paulo, Brazil

Correspondence to Dr Luciane Cruz Lopes; luslopes@terra.com.br

\section{SUMMARY}

Introduction Pain is one of the most common and most debilitating complaints among patients. It affects the individual, their relationship with friends and family, their ability to function at work, and their sociability. Acupuncture is one of the therapeutic resources for managing chronic pain. Given the variability of outcome measures in controlled randomised clinical trials on non-oncologicchronic pain (CRCT-NOCP), the Initiative in Methods, Measurements and Pain Assessment in Clinical Trials (IMMPACT) recommends six domains to be covered in evaluating the effectiveness of treatments for chronic pain.

Objective To check whether the methodological quality of outcome reporting in published trials has used IMMPACT recommendations in measuring CRCT-NOCP outcomes when acupuncture was used as a treatment.

Method This is a methodological study. We will systematically search for eligible studies in specific databases with a defined strategy. We will use the MeSHterms of 'acupuncture', 'chronic pain' and similar terms, without restrictions on idiom. Eligible studies will include those which are randomised and chose NOCP patients to be treated with acupuncture or control (sham acupuncture or no acupuncture), recruited after September 2004 , with $\geq 100$ patients. The measured outcomes are to be the presence of outcome domains recommended by IMMPACT, domains reported by the patient or clinician, tools used to measure such domains, as well as other features of the studies. We shall conduct a regression analysis to explore factors which can be associated with the presence of outcome domains according to IMMPACT recommendations.

Ethics and dissemination This survey will be submitted for presentation at congresses and for publication in a scientific journal. The findings obtained in this study will allow us to measure the quality of the evidence and provide greater transparency in decisions regarding the use of acupuncture as a viable alternative to managing chronic pain.

\section{INTRODUCTION}

Non-oncologic chronic pain (NOCP) is defined as a persisting painful feeling for more than some months, which may or may not be associated with trauma and illness. ${ }^{1}$ It is estimated that $18.9 \%$ of the world's population have chronic pain. It is one of the

\section{Strengths and limitations of this study}

- This is the first study to evaluate the outcome domains used in controlled randomised clinical trials (CRCT) using acupuncture as intervention for the treatment of non-oncologic chronic pain (NOCP).

- Acupuncture can be an effective therapy in chronic pain control, avoiding costly expenses associated with analgesic medication which generate dependence (opioids), or limiting adverse effects as in the case of non-steroidal anti-inflammatory drugs (gastric ulcer and cardiovascular events) that have a direct impact on the patient's life. Therefore, checking compliance with IMMPACT (Initiative in Methods, Measurements and Pain Assessment in Clinical Trials) recommendations in CRCT about NOCP may appraise the quality of the evidence and provide greater transparency in decisions regarding the use of acupuncture as a viable alternative in this clinical condition. It can also guide physicians in clinical practice decision making.

- The methods contain explicit eligibility criteria, comprehensive research, and a double independent selection, including independent appraisal of bias risk.

- Primary studies are probably limited in conception and outcome measures and thus they have high bias risk. Also, techniques or point categories used in acupuncture may be uncertain or varied in different studies.

- This enquiry has not received any specific sponsorship from any public, private or non-profit agency.

most common complaints among patients, affecting not only the subject in their individuality, but also in a general way. ${ }^{2}$

It is regarded as a health problem that consumes $22 \%$ of primary health appointments on average. In the USA, costs arising from pain medication are around US $\$ 17.8$ billion a year. ${ }^{3}$ In Canada the average monthly cost is Cd\$1462 for an individual with chronic pain on a waiting list, ${ }^{4}$ while in Portugal the cost is $€ 1883.30$ per adult. ${ }^{5}$ 
Acupuncture is one of the resources of the National Policy of Integrated and Supplementary Practices (NPISP) in Brazil. It is a possible therapy for managing chronic pain, reducing costs to the government and adverse effects for the patient. ${ }^{6}$ The WHO has launched a strategy for the period 2014-2023 to integrate acupuncture into traditional medicine, supplementing the health system in a safe, respectful, accessible and effective way. ${ }^{7}$

The search for international guidelines on the use of acupuncture for NOCP in the adult population results in few findings or in conflicting recommendations. Acupuncture is recommended as an adjunct to conventional treatment of NOCP, but only the US guidelines specify the moment in which it should be used within the conventional drug treatment flow. ${ }^{2-10}$

Among the policies we looked up, the recommendation of acupuncture for many painful conditions is based on low quality evidence due to the diversity in the methodology of CRCT. ${ }^{28-10}$ Also, such guidelines do not discriminate the power of the recommendation, except for those in Scotland and Canada. ${ }^{28}$

Even when of quality, CRCT with adequate randomisation and blinding may not provide the best approach for the development of a strong evidence base for managing pain, in case the outcomes and its tools are not adequate. ${ }^{11}$ Such limitations have been recognised internationally, leading to the development of the Initiative on Methods, Measurements and Pain Assessment in Clinical Trials (IMMPACT) in 2002.

The initiative gathered 27 experts from universities, governmental agencies and the pharmaceutical industry, who identified consensually a nucleus of six outcome domains that should be considered in CRCT for chronic pain. ${ }^{12}$ The outcome domains considered were: (1) pain; (2) physical function; (3) emotional state; (4) evaluation of the participants regarding improvement and satisfaction with treatment; (5) adverse symptoms; and (6) the participant's willingness; the first four domains were listed as the main ones. ${ }^{13}$

The establishment of a standard set of outcome domains in CRCT about chronic pain encourages researchers to consider chronic pain as a complex phenomenon which affects patients in multiple dimensions. It protects against the polarisation of selective outcomes, a common problem in all medical literature. It makes systematic reviews and meta-analyses easier, which allows researchers to generate more precise estimations of treatment effects due to the sharing of common outcomes of individual trials. ${ }^{14}$

Variability in outcome measures in CRCT about NOCP generates inaccuracies in the effectiveness of certain treatments. Although the recommendations of IMMPACT were published in 2003 and updated in 2008, there is no information on whether subsequent published clinical trials comply with the IMMPACT recommendations on their outcome measures.

The general aim of this project is to verify whether methodological quality of outcome reporting in published trials have used the IMMPACT recommendations in measuring CRCT-NOCP outcomes, which were executed as of September 2004 when acupuncture was used as a treatment.

\section{METHODS \\ Study design}

The study comprises a methodological survey of randomised clinical trials which used acupuncture for the treatment of chronic pain. The methodological survey is a type of study on method enquiry, with data collection from selected CRCT, not based on questionnaires but using systematic methods in its execution.

\section{Research question}

The question that guides this study was formulated using the Patient, Intervention, Comparison and Outcomes (PICO) strategy. In evidence-based practice, these four components are the fundamental elements of the research question and the construction of the question for the bibliographic search for evidence. An adequate research question allows the correct definition of what information (evidence) is necessary to solve the clinical research question, maximises the retrieval of evidence in the databases, focuses the scope of the research, and avoids unnecessary searches. ${ }^{15}$

Using the PICO strategy, the question of this survey was: randomised controlled trial (RCT) with individuals with NOCP (population) treated with acupuncture (intervention), where the comparator used was acupuncture sham or not acupuncture (comparison), and whether they reported the domains of IMMPACT recommendations (outcome).

\section{Reference sources and search}

All trials already included in CRCT-NOCP, published as of September 2004, were selected in the systematic review carried out by Vickers et al. ${ }^{16}$ Additional research will be performed in studies dating from January 2011 and 6 months before the systematic review of the comprehensive search date on the theme (considering delay in indexing) to nowadays. The search for eligible studies will be accomplished by systematic research of several databases, namely Lilacs, CINAHL, Embase, Medline, AMED, Web of Science, Clinical Trials and Cochrane Central Registry of Controlled Trials, with a defined search strategy, free of idiom restriction.

We shall combine the main terms 'Chronic Pain' and 'Acupuncture' indexed in the MeSH system. First, we will search the isolated terms and their synonyms, and then we will make a second search, combining and crossing the terms (see online supplementary appendix A and table 1).

We will verify the reference or citation list found in secondary studies to identify possibly eligible studies. When necessary, we shall contact the authors of the main studies to obtain further information. 


\section{Study eligibility criteria}

The eligibility criteria for this study will be the same as those adopted in the systematic review published in 2012.

\section{Design}

Controlled randomised clinical trials, whose patient recruitment occurred from September 2004 and in which the number of patients is $\geq 100$, will be included in the study.

\section{Clinical condition}

The studies will include patients aged 18 or older, with non-oncologic chronic pain. Eligible pain conditions are: osteoarthritis, chronic or recurrent headaches, specific and non-specific shoulder pain, and non-specific back or neck pain. For osteoarthritis or headaches, it will not be necessary to specify the duration of the pain, since both are of a chronic nature. For pain in the shoulder, back and neck, the pain episode should be at least 4 weeks in duration.

\section{Intervention}

The studies should include one group of patients treated with acupuncture, where acupuncture points or trigger points were stimulated with acupuncture needles, and another group where patients were treated with sham acupuncture or no acupuncture, and studies where the choice of blinding is unmistakable and adequate.

\section{Exclusion criteria}

Trials involving neck or back pain associated with specific clinical conditions (eg, fractures resulting from osteoporosis) will be excluded.

\section{Determination of eligibility}

Two reviewers, in pairs, will evaluate independently whether summaries and titles are in accordance with the eligibility criteria. Differences are to be resolved by consensus among all the reviewers. To assess the agreement of the selection we will use the Kappa Test, given that $\kappa$ values between 0.40 and 0.59 are to be considered weak agreement, between 0.60 and 0.74 medium agreement, and $\geq 0.75$ excellent agreement.

In order to exclude duplicate articles, one reviewer will analyse all the eligible articles and identify those which have one or more authors in common. In the case of duplicate publication, we will use the article with most complete data.

\section{Data extraction}

We will adopt an Excel spreadsheet for abstraction of the data, to be used by two reviewers separately. A third reviewer will check the Excel spreadsheet to ensure the coherence of the answers obtained among collaborators and use the consensus when necessary.

For articles published only in summary or for those with important information that is missing, we will look for complete information about methods and results by contacting the authors.
Two reviewers will be calibrated by the extraction of at least three articles, and then will perform the consensus, in pairs and independently. This procedure will occur until the reviewers are able to extract the data. The collected data will be: name of the first author, date of publication, country of origin, impact of the journal, recruitment date of the first participant, presence of outcome domains of IMMPACT, and the tools used for measuring the outcome domains, method of acupuncture, clinical condition of the patient, and duration of treatment. In addition, the study will check if fundamental outcomes are reported by the patient (ORP), if clinical outcomes are reported (COR), if the outcome was reported by a third person (ORT), or a combination of the items above.

The data will be recorded to be transferred to a statistical analysis programme later. A regression analysis will be conducted to explore factors that may be associated with the presence of outcome domains according to IMMPACT recommendations.

\section{Risk of bias}

A modified version of Cochrane for risk of bias will be used. ${ }^{1417}$ Reviewers will evaluate the risk of bias for each randomised trial independently, according to the following criteria: generation of random sequence; hiding of the choices; blinding of participants and professionals; blinding of outcome evaluators; whether outcomes were reported adequately; incomplete outcomes; selective outcome reporting and other sources of bias. Reviewers will attribute the answers 'definitely yes', 'probably yes', 'probably not' and 'definitely not' to each of the domains. ${ }^{18}$ Ultimately, 'definitely yes' and 'probably yes' will be attributed as low risk of bias, whereas 'definitely not' and 'probably not' will mean high risk of bias. Reviewers will solve divergences through discussion, and a third person will judge unsolved divergences.

\section{Definitions of IMMPACT outcome domain}

The four IMMPACT domains recommended in 2003 and 2008 which will be captured in this study are listed below, together with their definitions

1. Pain: Includes various aspects of pain evaluation (eg, intensity of pain, duration and frequency). The global evaluation of pain is a general assessment which examines how the pain changed during the treatment.

2. Physical function: refers to the participant's capacity to conduct their daily activities (eg, tasks, walks, trips and self-care), strength and resistance.

3. Emotional state: refers to the treatment associated with emotional anguish (eg, depression, anxiety, anger or irritability).

4. Patient's classification of improvement and satisfaction with the treatment: refers to the participant's feelings about the treatment (ie, whether they feel the positive features of the treatment surpass the negative ones). This domain overcomes pain classification only.

Thereafter, for each domain, the measurement method is quantized, that is, whether the pain was measured by 
Visual Analogue Scale (VAS) and/or Visual Numerical Scale (VAN), whether physical function was measured by multidimensional inventory to pain and/orinventory summary of the pain, whether the emotional state was measured by the Beck depression inventory and/ormood state profile, and whether the improvement in patient satisfaction was measured by the patient's overall impression of change. The correct applicability of the instrument will also be quantified (if the domain report was executed by the patient, clinician or third parties).

\section{Statistical analysis}

The statistical analyses carried out in this survey will aim to identify the factors associated with the change in reporting or adherence to the IMMPACT recommendations, in RCTs conducted since its publication.

The descriptive part includes year of publication, place of study, impact factor of the journal, and items of evaluation of methodological quality. These factors will be highlighted as they may influence the adherence of the IMMPACT recommendations. Afterwards, the frequency of measurement of pain, physical function, emotional state and patient satisfaction improvement will be described according to the IMMPACT recommendations.

Compliance with IMMPACT will be measured by the attendance of the four main domains. It is also planned to quantify the number of IMMPACT domains that will be served, in order to generate a score between $0-4$ points. The score will be presented as average, $\mathrm{SD}$, median and $\mathrm{IQR}$.

A score of 0 will be given when the study does not report any of the domains recommended by IMMPACT; score 1 when reporting only one of the recommended domains; score 2 when reporting two of the recommended domains; score 3 when reporting three of the recommended domains; and score 4 when reporting the four major domains recommended by IMMPACT.

The factors associated with compliance with the areas of IMMPACT will be investigated. For this, a logistic regression will be performed considering the domains of IMMPACT as dependent variables and the characteristics of the study as independent variables (year of publication, place of study, periodic impact factor, and items of methodological quality evaluation). For a good regression analysis, a minimum of 10 references is necessary, which will not be a problem since we will include previous systematic review (SR) studies. The results will be expressed in odds ratios with respective $95 \%$ confidence intervals (OR, 95\% CI).

Factors associated with the IMMPACT score will also be investigated. Depending on the data distribution, analysis of variance (ANOVA) or Kruskal-Wallis will be performed. All analyses will be 2-sided tests at a significance level of 0.05 .

All calculations will run in STATA 14.2.

\section{DISCUSSION}

Our survey will evaluate methodologically the outcomes of RCTs which used acupuncture for NOCP. We will check whether the methodological quality of outcome reporting in published trials have used IMMPACT recommendations in measuring CRCT-NOCP outcomes when acupuncture was used as a treatment. Our survey's outcomes will be significant for public health and for health professionals all over the world, mainly in Brazil.

Since the publication of IMMPACT, it is not known whether studies using acupuncture as an intervention for chronic pain follow IMMPACT's recommendations. Without consistent and more thorough standard outcome reports for patients in CRTC and NOCP, the authors of such studies will be unable to judge objectively the effects of acupuncture. The data compiled on the use of acupuncture will inform both patients and health professionals about its efficacy and safety. Therefore, multiprofessional care and decision-making based on evidence will be made easier.

This project aims at exploring some hypotheses to determine the use of IMMPACT recommendations on CRTC-NOCP. After the publication of IMMPACT orientations in August 2003 and later, in 2008, CRTC-NOCP which started recruiting participants as from September 2004 had better reports of main outcomes regarding IMMPACT domains versus journals with lower impact factors. The main domains were reported by the patient, by the clinician, by a third person or by a combination of these subjects.

Acknowledgements The authors thank Dr. Caio Guimarães for his expert advice.

Contributors $\mathrm{LCL}$ is the main researcher who led the writing of the manuscript. CCB and LGM are the project manager andco-researcher, who contributed to the writing and review of the manuscript. MTS is co-researcher, and contributed to the writing and review of the manuscript. All the authors have read and approved the final manuscript.

Competing interests None declared.

Provenance and peer review Not commissioned; externally peer reviewed. Data sharing statement № additional data are available.

Open Access This is an Open Access article distributed in accordance with the Creative Commons Attribution Non Commercial (CC BY-NC 4.0) license, which permits others to distribute, remix, adapt, build upon this work non-commercially, and license their derivative works on different terms, provided the original work is properly cited and the use is non-commercial. See: http://creativecommons.org/ licenses/by-nc/4.0/

(C) Article author(s) (or their employer(s) unless otherwise stated in the text of the article) 2017. All rights reserved. No commercial use is permitted unless otherwise expressly granted.

\section{REFERENCES}

1. Ontario RNA. Assessment and management of pain. 3rd ed. Dez/2013 ed. Rockville MD: Agency for Healthcare Research and Quality (AHRQ), 2013.

2. SIGN. Management of chronic pain. A national clinical guideline. Secondary management of chronic pain. A national clinical guideline 2013. http://www.guideline.gov/content.aspx?id=47707\& search=chronic +pain

3. Rasu RS, Vouthy K, Crowl AN, et al. Cost of pain medication to treat adult patients with nonmalignant chronic pain in the United States. J Manag Care Spec Pharm 2014;20:921-8.

4. Guerriere DN, Choinière M, Dion D, et al. The Canadian STOPPAIN project - Part 2: What is the cost of pain for patients on waitlists of multidisciplinary pain treatment facilities? Can $J$ Anaesth 2010;57:549-58.

5. Azevedo LF, Costa-Pereira A, Mendonça L, et al. The economic impact of chronic pain: a nationwide population-based 
cost-of-illness study in Portugal. Eur $\mathrm{J}$ Health Econ 2016; 17:87-98

6. Brasil. Política Nacional de Práticas Integrativas e Complementares no SUS. Ministério da Saúde 2006 DOU - secão 1.

7. Salud OMdl. Estrategia de la OMS sobre medicina tradicional 20142023, 2013.

8. Ontario CPSO. Evidence-based recommendations for medical management of chronic non-malignant pain. CPSO, 2000.

9. American Society of Anesthesiologists Task Force on Chronic Pain Management, American Society of Regional Anesthesia and Pain Medicine. Practice guidelines for chronic pain management: an updated report by the American Society of Anesthesiologists Task Force on Chronic Pain Management and the American Society of Regional Anesthesia and Pain Medicine. Anesthesiology 2010;112:810-33.

10. National Guideline. Assessment and management of chronic pain. secondary assessment and management of chronic. Pain $2011 \mathrm{http}: / / \mathrm{www}$. guideline.gov/content.aspx?id=47646\&search= assessment+and+management+of+chronic+pain
11. Dworkin RH, Turk DC, Peirce-Sandner S, et al. Research design considerations for confirmatory chronic pain clinical trials: IMMPACT recommendations. Pain 2010;149:177-93.

12. Turk DC, Dworkin RH, Allen RR, et al. Core outcome domains for chronic pain clinical trials: IMMPACT recommendations. Pain 2003;106:337-45.

13. Dworkin $\mathrm{RH}$, Turk DC, Farrar JT, et al. Core outcome measures for chronic pain clinical trials: IMMPACT recommendations. Pain 2005;113:9-19.

14. Higgins JP. Cochrane handbook for systematic reviews of interventions version [5.1.0], 2011.

15. da Costa Santos CM, de Mattos Pimenta CA, Nobre MR. The PICO strategy for the research question construction and evidence search. Rev Lat Am Enfermagem 2007;15:508-11.

16. Vickers AJ, Cronin AM, Maschino AC, et al. . Acupuncture for chronic pain: individual patient data meta-analysis. Arch Intern Med 2012;172:1444-53.

17. Akl EA, Sun X, Busse JW, et al. Specific instructions for estimating unclearly reported blinding status in randomized trials were reliable and valid. J Clin Epidemiol 2012;65:262-7.

18. Busse J, Guyatt G. Modification of Cochrane Tool to assess risk of bias in randomized trials, 2013. 